\title{
Counting Singular Matrices with Primitive Row Vectors
}

\author{
Igor Wigman \\ School of Mathematical Sciences \\ Tel Aviv University \\ Tel Aviv 69978, Israel
}

November 21, 2018

\begin{abstract}
We solve an asymptotic problem in the geometry of numbers, where we count the number of singular $n \times n$ matrices where row vectors are primitive and of length at most $\mathrm{T}$. Without the constraint of primitivity, the problem was solved by Y. Katznelson. We show that as $T \rightarrow \infty$, the number is asymptotic to $\frac{(n-1) u_{n}}{\zeta(n) \zeta(n-1)^{n}} T^{n^{2}-n} \log (T)$ for $n \geq 3$. The 3 -dimensional case is the most problematic and we need to invoke an equidistribution theorem due to W. Schmidt.
\end{abstract}

Keywords: singular matrices, primitive vectors, lattices, equidistribution theorem, asymptotics.

AMS classification numbers: $11 \mathrm{H} 06$.

\section{Introduction}

\section{1}

A basic problem in geometry of numbers is counting integer matrices with certain additional properties. In this paper we will solve a new counting problem of this kind. Let us consider the set of singular $n \times n$ matrices with integer entries. We are interested in the question how many among these matrices have primitive row vectors, that is each row is not a nontrivial multiple of an integer vector. We count the matrices according to the maximal

allowed Euclidean length of the rows. Without the constraint of primitivity 
the problem of counting such matrices was solved by Y. Katznelson [K]. We will find that for $n \geq 3$ a positive proportion of integer singular matrices have all rows primitive.

Let $P N_{n}(T)$ be the counting function of the set $P M_{n}(T)$ of $n \times n$ singular integer matrices, all of whose rows are primitive and whose Euclidian length is at most $T$. That is, let

$$
P M_{n}(T)=\left\{M \in M_{n}(\mathbb{Z}): \operatorname{det}(M)=0 \text {, primitive rows } v_{i} \in \mathbb{Z}^{n},\left|v_{i}\right| \leq T\right\},
$$

and set $P N_{n}(T)=\left|P M_{n}(T)\right|$. In this paper we will determine the asymptotic behaviour of $P N_{n}$, as $T \rightarrow \infty$.

Define a similar counting function $N_{n}(T)$, where $N_{n}$ counts $n \times n$ integer matrices $\mathrm{M}$ with rows of length $\leq T$, that is $N_{n}(T)=\left|M_{n}(T)\right|$, where

$$
M_{n}(T)=\left\{M \in M_{n}(\mathbb{Z}): \operatorname{det}(M)=0, \text { rows } v_{i},\left|v_{i}\right| \leq T\right\} .
$$

Y. Katznelson [K] showed that for $n \geq 3$,

$$
N_{n}(T)=\frac{(n-1) u_{n}}{\zeta(n)} T^{n^{2}-n} \log (T)+O\left(T^{n^{2}-n}\right),
$$

where the constant in the $O$-notation depends only on $n$. Recall that $n !$ denotes the product of integers $\leq n$ of the same parity as $n$. The constant $u_{n}$ is given by:

$$
u_{n}=\left\{\begin{array}{ll}
\frac{n}{2}\left(\frac{2(2 \pi)^{m-1}}{(2 m-1) ! !}\right)^{n} \cdot \frac{\pi^{m}}{m !}, & n=2 m \\
\frac{n}{2}\left(\frac{\pi^{m}}{m !}\right)^{n} \cdot \frac{2(2 \pi)^{m}}{(2 m+1) ! !}, & n=2 m+1
\end{array} .\right.
$$

Trivially, $P N_{n}(T) \leq N_{n}(T)$, and thus $P N_{n}(T) \ll T^{n^{2}-n} \log (T)$. Moreover, $P N_{n}(T) \gg T^{n^{2}-n}$, since we can consider, for example, only matrices $\mathrm{M}$ with primitive rows $v_{i}$, which satisfy $v_{n}=v_{1}$ and $\left|v_{i}\right| \leq T$. A random vector in $\mathbb{Z}^{n}$ is primitive with a positive probability, that is, the number of primitive vectors whose length is at most $T$, is $\gg T^{n}$. The number of such matrices is obviously $\gg\left(T^{n}\right)^{n-1}=T^{n^{2}-n}$. Combining the observations of this paragraph, we conclude that $T^{n^{2}-n} \ll P N_{n}(T) \ll T^{n^{2}-n} \log (T)$.

For $n=2$ an elementary argument shows that

$$
P N_{2}(T)=\frac{2 \pi}{\zeta(2)} T^{2}+O(T)
$$

Our main result is:

Theorem 1. (i). For $n \geq 4$ we have

$$
P N_{n}(T)=\frac{(n-1) u_{n}}{\zeta(n) \zeta(n-1)^{n}} T^{n^{2}-n} \log (T)+O\left(T^{n^{2}-n}\right) .
$$


(ii). For $n=3$ we have

$$
P N_{3}(T)=\frac{2 u_{3}}{\zeta(3) \zeta(2)^{3}} T^{6} \log (T)+O\left(T^{6} \log \log (T)\right) .
$$

\section{2}

Another way to treat our problem is to consider it as a counting problem of rational points with bounded height on a projective variety, see [FMT].

Let $V \subset \underbrace{\mathbb{P}^{n-1} \times \ldots \times \mathbb{P}^{n-1}}_{n \text { times }}$ be the projective variety where the determinant vanishes. The height of a point $X \in \mathbb{P}^{n-1}(\mathbb{Q})$ is defined by

$$
H^{\prime}(X)=|\tilde{X}|,
$$

where $\tilde{X}$ is a primitive integral point in $\mathbb{Z}^{n}$ representing $\mathrm{X}$ and $|\cdot|$ is the standard Euclidian norm on $\mathbb{R}^{n}$. Now, for $Y=\left(Y_{1}, \cdots, Y_{n}\right) \in V$, define the height

$$
H(Y)=\max _{1 \leq i \leq n} H^{\prime}\left(Y_{i}\right)
$$

Then $P N_{n}(T)$ is the number of points $Y \in V$ of height $H(Y) \leq T$.

\section{3}

We will present now the main idea in the case $n=3$. If $\mathrm{M}$ is an integer singular matrix, then all the rows of $\mathrm{M}$ lie in a 2-dimensional lattice $\Lambda \subset \mathbb{Z}^{3}$. Thus we should count triples of vectors lying in a 2-dimensional sublattice of $\mathbb{Z}^{3}$ and sum over all such lattices.

For a primitive $\lambda \in \mathbb{Z}^{3}$ we define a 2-dimensional lattice $L_{\lambda}=\{v \in$ $\left.\mathbb{Z}^{3}: v \perp \lambda\right\}=\lambda^{\perp}$. Denote the subset of all primitive points in $L_{\lambda}, P L_{\lambda}=$ \{primitive $\left.v \in L_{\lambda}\right\}$ and $P L_{\lambda}(T)=\left\{v \in P L_{\lambda}:|v| \leq T\right\}$. Moreover, denote $P A_{\lambda}=\left\{M \in M_{3}(\mathbb{Z}):\right.$ rows in $\left.P L_{\lambda}\right\}$, and $P A_{\lambda}(T)=\left\{M \in P A_{\lambda}: \mid\right.$ rows $\mid \leq$ $T\}$. Thus $\left|P A_{\lambda}(T)\right|=\left|P L_{\lambda}(T)\right|^{3}$. For $\lambda \neq \lambda^{\prime}$ the intersection $L_{\lambda} \cap L_{\lambda^{\prime}}$ is a set of integer points on a line, and thus $\left|P A_{\lambda} \cap P A_{\lambda^{\prime}}\right| \leq 2^{3}=8$. It can be shown that the contribution of such intersections is negligible, and that

$$
P N_{n}(T) \sim \sum_{|\lambda| \ll T^{2}}^{\prime}\left|P L_{\lambda}(T)\right|^{3}
$$

where the last sum is over primitive $\lambda \in \mathbb{Z}^{3}$, such that $L_{\lambda}$ is "bounded by T" (see section 5). Now

$$
P L_{\lambda}(T)=\frac{v_{2}}{\zeta(2)|\lambda|} T^{2}+O\left(\frac{T \log (T)}{\left|\lambda_{1}\right|}\right)
$$


(see section (3), and summing the cube of the main term of $P L_{\lambda}(T)$ will give the result.

A complication in dimension 3 is that for some of the lattices $L_{\lambda}$ in the sum (4), the error term in (5) is asymptotically greater than the main term. Such a phenomenon does not happen for higher dimensions. In order to show that this phenomenon is $\underline{\text { rare }}$ and the contribution of such lattices is negligible, we will use an equidistribution theorem of Wolfgang Schmidt [SCD] (see theorem [2).

\subsection{Contents:}

We will use some known results of counting integer points in $\mathbb{Z}^{2}$, or more generally, counting points of a sublattice of $\mathbb{Z}^{n}$, as well as counting primitive points in such a sublattice. We will give some basic background on lattices in section 2 and some facts concerning counting lattice points will be given in section [3, The goal of sections 4 and 5 is to prove cases (ii) and (i) of theorem 1 respectively.

\subsection{Acknowledgement:}

This work was carried out as part of the author's M.Sc. thesis at Tel Aviv University, under the supervision of Prof. Zeev Rudnick. The author was supported in part by the Israel Science Foundation, founded by the Israel Academy of Science and Humanities.

\section{Background on lattices}

In this section we will give some basic facts which deal with sublattices of $\mathbb{Z}^{n}$. For general background see SGL].

Definition: Let $\Lambda$ be a lattice. A basis of $\Lambda,\left\{\lambda_{1}, \lambda_{2}, \ldots \lambda_{m}\right\}$, such that the product of the lengths of the vectors in it is minimized is called reduced. For such a basis we have:

$$
\left|\lambda_{1}\right| \cdot\left|\lambda_{2}\right| \cdot \ldots \cdot\left|\lambda_{m}\right| \ll \gg \operatorname{det}(\Lambda) .
$$

A basis that satisfies the last inequality has properties similar to a reduced one.

We say that $\Lambda$ is bounded by $T$, if it has a reduced basis consisting of vectors of length at most $T$. If a $k$-dimensional lattice has $k$ linearly independent vectors, all of length at most $T$, it follows that this lattice is bounded by $c T$, 
for some constant $c$ that depends only on the dimension $k$. In that case we will treat it just as if it was bounded by $T$, since it will affect only some constants in our upper bounds not affecting the asymptotic behavior.

Also, for a lattice $\Lambda$, we will denote

$$
N_{\Lambda}(T)=|\{v \in \Lambda:|v| \leq T\}|
$$

as well as

$$
P_{\Lambda}(T)=\mid\{v \in \Lambda:|v| \leq T, \quad v \text { primitive }\} \mid .
$$

An $m$-dimensional lattice $\Lambda \subset \mathbb{Z}^{n}$ is called primitive if there is no $m$ dimensional lattice $\Lambda^{*}$ properly containing $\Lambda$. In particular, each vector in any basis of a primitive lattice is a primitive vector (the converse is not necessarily true). The orthogonal lattice $\Lambda^{\perp}$ of $\Lambda$ consists of all vectors $v \in \mathbb{Z}^{n}$, such that $v \cdot u=0$ for all $u \in \Lambda$. It is a primitive integral lattice of dimension $n-m$.

If $\Lambda$ is a primitive lattice, then $\left(\Lambda^{\perp}\right)^{\perp}=\Lambda$. Also, in this case, it was shown in $\mathrm{SCH}]$ (chapter 1, formula (4)), that

$$
\operatorname{det}(\Lambda)=\operatorname{det}\left(\Lambda^{\perp}\right)
$$

\section{Counting lattice points}

The goal of this section will be to give some expressions for the number of integer points in a lattice, as well as estimations for the error terms of these expressions, which correspond to primitive lattices.

The next lemma is a basic one, which could be found in different variations in the literature, see e.g. [SCH], lemma 2.

Lemma 1. Let $\Lambda \subset \mathbb{R}^{n}$ be an m-dimensional lattice, and let $\left\{\lambda_{1}, \ldots, \lambda_{m}\right\}$ be a reduced basis for $\Lambda$, sorted in increasing order of their norms. Denote $\left|\lambda_{i}\right|=\mu_{i}$ for $1 \leq i \leq m$. Let $\beta \subset \mathbb{R}^{m}$ be an $m$-dimensional convex body containing $m$ linearly independent vectors of $\Lambda$, then

$$
|\Lambda \cap \beta|=\frac{\operatorname{vol}(\beta)}{\operatorname{det}(\Lambda)}+O\left(\frac{\operatorname{vol}(\partial \beta)}{\mu_{1} \cdot \ldots \cdot \mu_{m-1}}\right)
$$

(the $\mu^{\prime} s$ in the denominator are all except for the greatest one).

Let $v_{n}$ be the volume of the standard $n$-dimensional unit ball, that is

$$
v_{n}=\left\{\begin{array}{cl}
\frac{\pi^{m}}{m !}, & n=2 m \\
\frac{2(2 \pi)^{m}}{(2 m+1) ! !}, & n=2 m+1
\end{array} .\right.
$$


Lemma 2. Let $\Lambda \subset \mathbb{Z}^{n}$ be an $(n-1)$-dimensional primitive lattice which is bounded by $T$, for $n \geq 3$. Let $\left\{\lambda_{1}, \lambda_{2}, \ldots, \lambda_{n-1}\right\}$, be a reduced basis of $\Lambda$. Then:

(i). For $n \geq 4$ we have

$$
P_{\Lambda}(T)=\frac{v_{n-1}}{\zeta(n-1) \operatorname{det}(\Lambda)} T^{n-1}+O\left(\frac{T^{n-2}}{\left|\lambda_{1}\right| \cdot\left|\lambda_{2}\right| \cdot \ldots \cdot\left|\lambda_{n-2}\right|}\right) .
$$

(ii). For $n=3$ we have

$$
P_{\Lambda}(T)=\frac{v_{2}}{\zeta(2) \operatorname{det}(\Lambda)} T^{2}+O\left(\frac{T \log T}{\left|\lambda_{1}\right|}\right) .
$$

Proof. Since $\Lambda$ is primitive, every vector is a [possibly trivial] integer multiple of a primitive vector $\underline{\text { in } \Lambda}$, and thus $N_{\Lambda}(T)=\sum_{k=1}^{\lfloor T\rfloor} P_{\Lambda}\left(\frac{T}{k}\right)$, hence by Moebius inversion

$$
P_{\Lambda}(T)=\sum_{k=1}^{\lfloor T\rfloor} \mu(k) \cdot N_{\Lambda}\left(\frac{T}{k}\right) .
$$

Using on the last expression the result of lemma 1 where $m=n-1$, and $\beta$ is the $(n-1)$-dimensional ball on the $(n-1)$-dimensional hyper-plane spanned by $\Lambda$, we get

$$
\begin{aligned}
P_{\Lambda}(T) & =\sum_{k=1}^{\lfloor T\rfloor} \mu(k) \cdot\left(\frac{v_{n-1}}{\operatorname{det}(\Lambda)}\left(\frac{T}{k}\right)^{n-1}+O\left(\frac{T^{n-2}}{k^{n-2} \cdot\left|\lambda_{1}\right| \cdot\left|\lambda_{2}\right| \cdot \ldots \cdot\left|\lambda_{n-2}\right|}\right)\right) \\
& =\frac{v_{n-1} T^{n-1}}{\operatorname{det} \Lambda} \sum_{k=1}^{\lfloor T\rfloor}\left(\mu(k) k^{-(n-1)}+\epsilon_{k}^{\prime}(T)\right)=\frac{v_{n-1}}{\zeta(n-1) \operatorname{det}(\Lambda)} T^{n-1}+\epsilon(T) .
\end{aligned}
$$

with error term $\epsilon(T)$ given by

$$
\begin{aligned}
\epsilon(T) & =\sum_{k=1}^{\lfloor T\rfloor} O\left(\frac{T^{n-2}}{k^{n-2} \cdot\left|\lambda_{1}\right| \cdot\left|\lambda_{2}\right| \cdot \ldots \cdot\left|\lambda_{n-2}\right|}\right) \\
& +\frac{v_{n-1} \cdot T^{n-1}}{\operatorname{det} \Lambda}\left(\sum_{k=1}^{\lfloor T\rfloor} \mu(k) k^{-(n-1)}-\frac{1}{\zeta(n-1)}\right) .
\end{aligned}
$$


Thus, since $|\mu(n)| \leq 1$ for every $n \in N$,

$$
\begin{aligned}
|\epsilon(T)| & \ll \frac{1}{\left|\lambda_{1}\right| \cdot\left|\lambda_{2}\right| \cdot \ldots \cdot\left|\lambda_{n-2}\right|}\left(\sum_{k=1}^{\lfloor T\rfloor} \frac{T^{n-2}}{k^{n-2}}+T^{n-1} \cdot\left|\sum_{k=\lfloor T\rfloor+1}^{\infty} \mu(k) k^{-(n-1)}\right|\right) \\
& \ll \frac{1}{\left|\lambda_{1}\right| \cdot\left|\lambda_{2}\right| \cdot \ldots \cdot\left|\lambda_{n-2}\right|}\left(T^{n-2} \sum_{k=1}^{\lfloor T\rfloor} \frac{1}{k^{n-2}}+T^{n-1} \cdot \frac{1}{T^{n-2}}\right)
\end{aligned}
$$

We also used here the fact that $\left|\lambda_{1}\right| \cdot\left|\lambda_{2}\right| \cdot \ldots \cdot\left|\lambda_{n-2}\right| \ll \operatorname{det}(\Lambda)$. Now in order to obtain case (i) of the lemma use the convergence of the series $\sum_{k=1}^{\infty} \frac{1}{k^{n-2}}$ for $n \geq 4$. We use $\sum_{k=1}^{\lfloor T\rfloor} \frac{1}{k} \ll \log (T)$ in order to prove the other case of the lemma.

\section{The case $n=3$}

In this section we will prove case (ii) of theorem 11. The computation of the main term is also valid in the case $n \geq 4$, while for $n=3$ we should be more delicate in order to obtain the appropriate error term. Thus every lemma which is to be used in section [5] will be stated for general $n$ in the current section.

The main difficulty in this case is that the error term for estimating $P_{\Lambda}$ (see lemma 2, case (ii) could be asymptotically greater than the main term itself. In order to show that such lattices are rare, and thus their contribution to the error term is asymptotically negligible, we will need an equidistribution result of W. Schmidt [SCD] for the space of lattices.

Suppose $M$ is a singular matrix. This means that there exists a vector $0 \neq \lambda \in \mathbb{Z}^{n}$, such that all rows of $M$ are orthogonal to $\lambda$. Thus all the rows of $M$ lie in a $(n-1)$-dimensional lattice $\lambda^{\perp}$. Since multiplying $\lambda$ by a constant does not affect this property we can assume that $\lambda$ is primitive. Our basic idea is to sum the number of $n$-tuples of primitive vectors with bounded length lying in $\Lambda$, where $\Lambda \subset \mathbb{Z}^{n}$ runs over all such lattices. We will see that we can limit the sum to a finite number of such lattices.

Let $\lambda \in \mathbb{Z}^{n}$ be primitive. Recall that $\lambda^{\perp}$ denotes the $(n-1)$-dimensional orthogonal dual lattice to $\lambda$, that is the primitive $(n-1)$-dimensional lattice in $\mathbb{Z}^{n}$ which consists of all vectors in $\mathbb{Z}^{n}$ which are orthogonal to $\lambda$. Our discussion leads to the following definitions: denote $L_{\lambda}=\lambda^{\perp}$, 


$$
\begin{aligned}
L_{\lambda}(T) & =\left\{v \in L_{\lambda}:|v| \leq T\right\}, \\
P L_{\lambda} & =\left\{\text { primitive } v \in L_{\lambda}\right\}, \\
P L_{\lambda}(T) & =P L_{\lambda} \cap L_{\lambda}(T),
\end{aligned}
$$

Given $\lambda$ denote by $A_{\lambda}$ the set of matrices whose rows lie in $L_{\lambda}$ :

$$
A_{\lambda}=\left\{M \in M_{n}(\mathbb{Z}): M \cdot \lambda=0\right\}=\underbrace{L_{\lambda} \oplus L_{\lambda} \oplus \ldots \oplus L_{\lambda}}_{n \text { times }},
$$

Also denote by $P A_{\lambda}$ the subset of matrices in $A_{\lambda}$, whose rows are primitive. Obviously,

$$
P A_{\lambda}=\underbrace{P L_{\lambda} \oplus P L_{\lambda} \oplus \ldots \oplus P L_{\lambda}}_{n \text { times }}
$$

Denoting by $A_{\lambda}(T)$ and $P A_{\lambda}(T)$ the set of matrices in $A_{\lambda}$ with (primitive) rows in $L_{\lambda}$ of length $\leq T$, we clearly have:

$$
A_{\lambda}(T)=L_{\lambda}(T)^{n}
$$

and

$$
P A_{\lambda}(T)=P L_{\lambda}(T)^{n} .
$$

The next lemma connects between the terms just defined with our problem.

Lemma $3\left([\mathbf{K}]\right.$, Lemma 4). Let $X \in M_{n}(T)$. Then there is a primitive $\lambda \in \mathbb{Z}^{n}$, such that $A_{\lambda}$ is bounded by $T$ and $X \in A_{\lambda}$.

Remarks:

- As it were mentioned, "bounded by $T$ " means bounded by $c_{n} T$, where the constant $c_{n}>0$ depends only on $n$. We will see that $c_{n}$ doesn't affect our computations, so we will ignore it everywhere except the computation of the main term.

- If $A_{\lambda}$ is bounded by $T$ then $|\lambda|^{n}=\operatorname{det}\left(A_{\lambda}\right) \ll T^{n^{2}-n}$ by (6) and (17), and thus $|\lambda| \ll T^{n-1}$. The converse is not true, since there are primitive vectors $\lambda \in \mathbb{Z}^{n}$, such that $|\lambda| \leq T^{n-1}$, but $\overline{A_{\lambda}}$ is not bounded by $T$. We will call such vectors "bad"; they will only have a minor influence on the asymptotics.

- In every case we will deal with reduced basis, the vectors will be ordered in increasing order of their norms, unless specified otherwise.

We may conclude from lemma 3, that $M_{n}(T)=\underset{|\lambda| \ll T^{n-1}}{\bigcup_{\lambda}^{\prime \prime}} A_{\lambda}(T)$ and also

$$
P M_{n}(T)=\bigcup_{|\lambda| \ll T^{n-1}}^{\prime \prime} P A_{\lambda}(T) .
$$


Here and everywhere in this paper, we use $\bigcup^{\prime \prime}$ and $\sum^{\prime \prime}$ to denote a union/sum over primitive vectors $\lambda \in \mathbb{Z}^{n}$, for which $\lambda^{\perp}$ is T-bounded. Analogously, $\bigcup^{\prime}$ and $\sum^{\prime}$ will denote a union/sum over primitive vectors not saying anything about the orthogonal dual.

It is natural to relate the cardinality of the left side of (10) to sum of the cardinalities of the right side:

$$
\begin{aligned}
P N_{n}(T) & =\frac{1}{2} \cdot \sum_{|\lambda| \ll T^{n-1}}^{\prime \prime}\left|P A_{\lambda}(T)\right|+\epsilon_{1}^{\prime}(T) \\
& =\frac{1}{2} \cdot \sum_{|\lambda| \ll T^{n-1}}^{\prime}\left|P A_{\lambda}(T)\right|+\epsilon_{1}^{\prime}(T)+\epsilon_{3}^{\prime}(T)
\end{aligned}
$$

The factor of $\frac{1}{2}$ is due to the fact that $P A_{\lambda}=P A_{-\lambda}$; the terms $\epsilon_{1}^{\prime}(T)$ and $\epsilon_{3}^{\prime}(T)$ are the error terms which implied by the intersections of $P A_{\lambda}$ for different $\lambda$ and the contribution of the so called "bad" vectors respectively. (A "bad" vector is a primitive vector $\lambda$, with $|\lambda| \ll T^{n-1}$ such that $\lambda^{\perp}$ is not bounded by $\mathrm{T}$ ), which do not allow us to get an estimate of the primitive vectors contained within it. However $\left|\epsilon_{1}^{\prime}(T)\right| \leq\left|\epsilon_{1}(T)\right|$ and $\left|\epsilon_{3}^{\prime}(T)\right| \leq\left|\epsilon_{3}(T)\right|$, where $\epsilon_{1}, \epsilon_{3}$ are the analogous error terms in the case of the problem solved in [K], which were shown to be $O\left(T^{n^{2}-n}\right)$ ( [K] pages 130-133). Thus:

$$
\begin{aligned}
P N_{n}(T) & =\frac{1}{2} \sum_{|\lambda| \ll T^{n-1}}{ }^{\prime}\left|P A_{\lambda}(T)\right|+O\left(T^{n^{2}-n}\right) \\
& =\frac{1}{2} \sum_{|\lambda| \ll T^{n-1}}{ }^{\prime}\left|P L_{\lambda}(T)\right|^{n}+O\left(T^{n^{2}-n}\right)
\end{aligned}
$$

For $n=3$, we would like to demonstrate how we can achieve the bound for $\epsilon_{1}^{\prime}$, since in our case it is quite simple. Indeed, the only matrices in $P A_{\lambda} \cap P A_{\lambda^{\prime}}$ with primitive $\lambda \neq \pm \lambda^{\prime}$ are of the form $\left(\begin{array}{c} \pm v \\ \pm v \\ \pm v\end{array}\right)$ with primitive $v \in \mathbb{Z}^{3}$. Conversely, given a primitive $v \in \mathbb{Z}^{3}$, its contribution to the sum in (11) is $8 P N_{v^{\perp}}\left(c_{3} T^{2}\right)$, where the 8 factor is the number of all possible signs of the 3 rows. Hence:

$$
\left|\epsilon_{1}^{\prime}(T)\right| \ll \sum_{|v| \leq T} P N_{v^{\perp}}\left(T^{2}\right) \leq \sum_{|v| \leq T} N_{v^{\perp}}\left(T^{2}\right) \ll \sum_{|v| \leq T} \frac{T^{4}}{|v|},
$$

Now $\sum_{|v| \leq T} \frac{1}{|v|} \ll T^{2}$ where the last equality is due to summation by parts, making use of the fact that $\mid\left\{\right.$ primitive $v \in \mathbb{Z}^{3}$ with $\left.|v| \leq T\right\} \mid \ll T^{3}$. Thus $\left|\epsilon_{1}^{\prime}(T)\right| \ll T^{6}$. 
At this point we would like to substitute the result of lemma 2 into (11). This is exactly what we are going to do in case $n \geq 4$ (see section 5 ). However, for $n=3$, the error term could be asymptotically greater than the main term. In order to overcome this difficulty, we will first reduce the last sum to "convenient" lattices, that is those with not too big determinant (lemma 4) and where the norms of vectors in a reduced basis do not differ too much (lemma 51). Corollary 1 will show that for such lattices the error term is negligible relative to the corresponding main term.

We will adapt the following notations:

Notations: For a $(n-1)$-dimensional lattice $\Lambda \subset \mathbb{Z}^{n}$, we will denote the main term of $\left(P_{\Lambda}(T)\right)^{n}$ as well as the corresponding error term:

$$
c(T, \Lambda)=\frac{v_{n-1}^{n}}{(\operatorname{det}(\Lambda))^{n} \zeta(n-1)^{n}} T^{n^{2}-n}, \quad \epsilon(T, \Lambda)=\left(P_{\Lambda}(T)\right)^{n}-c(T, \Lambda) .
$$

Moreover, for a vector $\lambda \in \mathbb{Z}^{n}$ denote

$$
c(T, \lambda)=c\left(T, \lambda^{\perp}\right), \quad \epsilon(T, \lambda)=\epsilon\left(T, \lambda^{\perp}\right) .
$$

Lemma 4. For any constant $A>0$, the following estimate holds:

$$
\sum_{\frac{T^{2}}{(\log T)^{A}}<\operatorname{det}(\Lambda) \leq T^{2}} P_{\Lambda}(T)^{3} \ll T^{6} \log \log T,
$$

where the sum is over primitive T-bounded lattices $\Lambda \subset \mathbb{Z}^{3}$.

Proof. We will use here the trivial inequality $P_{\Lambda}(T) \leq N_{\Lambda}(T)$. Now, from lemma 1. $N_{\Lambda}(T)=\frac{\pi T^{2}}{\operatorname{det}(\Lambda)}+O\left(\frac{T}{\left|\lambda_{1}\right|}\right) \ll \frac{T^{2}}{\operatorname{det}(\Lambda)}$, where $\lambda_{1}=\lambda_{1}(\Lambda)$ is the shortest vector in a reduced basis of $\Lambda$ (that is, the shortest nontrivial vector in $\Lambda$ ). The last inequality is due to $\Lambda$ being bounded by $T$, since it implies $\frac{T^{2}}{\operatorname{det}(\Lambda)} \gg$ $\frac{T^{2}}{\left|\lambda_{1}\right| \cdot\left|\lambda_{2}\right|}=\frac{T}{\left|\lambda_{1}\right|} \cdot \frac{T}{\left|\lambda_{2}\right|} \geq \frac{T}{\left|\lambda_{1}\right|}$. We will denote by $n(r)$ the number of primitive two-dimensional lattices $\Lambda \subset \mathbb{Z}^{3}$ with $\operatorname{det}(\Lambda)=r$ and $N(t)=\sum_{r=1}^{\lfloor t\rfloor} n(r)$. Then

$$
N(t) \ll t^{3},
$$

since such $\Lambda$ are determined by a primitive vector $\pm \lambda$ orthogonal to it with 
$|\lambda|=\operatorname{det}(\Lambda) \leq t$; the number of such vectors is $\ll t^{3}$. Thus

$$
\begin{aligned}
& \sum_{\frac{T^{2}}{(\log T)^{A}}<\operatorname{det}(\Lambda) \leq T^{2}}\left(P_{\Lambda}(T)\right)^{3} \ll T^{6} \sum_{\frac{T^{2}}{(\log T)^{A}}<\operatorname{det}(\Lambda) \leq T^{2}} \frac{1}{\operatorname{det}(\Lambda)^{3}} \\
& \leq T^{6} \sum_{r=\left\lceil\frac{T^{2}}{(\log T)^{A}}\right\rceil}^{T^{2}} \frac{n(r)}{r^{3}} \ll T^{6}\left[\left.\frac{N(t)}{t^{3}}\right|_{\frac{T^{2}}{(\log T)^{A}}} ^{T^{2}}+\int_{\frac{T^{2}}{(\log T)^{A}}}^{T^{2}} \frac{N(t)}{t^{4}} d t\right] \\
& \ll T^{6} \cdot \log \log T .
\end{aligned}
$$

We used here summation by parts, substituting (14) to get an estimate for $N(t)$ in order to obtain the last inequality. This concludes the proof of the lemma. It should be noted, that in addition to what was originally stated, we proved here also the following inequality:

$$
\sum_{\frac{T^{2}}{(\log (T))^{A}}<\operatorname{det}(\Lambda) \leq T^{2}} c(T, \Lambda) \ll T^{6} \log (\log (T)),
$$

where $c(T, \Lambda)$ is as in (12).

We will need the following theorem, which is special case of theorem 5 from $[\mathrm{SCD}$.

Theorem 2. For $a \geq 1$ let $N(a, T)$ be the number of lattices $\Lambda \subset \mathbb{Z}^{3}$ with successive minima, $\left\{\mu_{1}, \mu_{2}\right\}$ which satisfy $\frac{\mu_{2}}{\mu_{1}} \geq a$, and $d(\Lambda) \leq T$, then:

$$
N(a, T)=\text { const } \cdot \arcsin \left(\frac{1}{2 a}\right) T^{3}+O\left(a^{-\frac{1}{2}} \cdot T^{\frac{5}{2}}\right)
$$

We will use Theorem 2 in order to prove the following lemma:

Lemma 5. For any constants $A>0, B>1$, the following estimate holds:

$$
\sum_{\substack{\operatorname{det}(\Lambda)<\frac{T^{2}}{(\log T)^{A}} \\ \frac{\left|\lambda_{2}\right|}{\left|\lambda_{1}\right|}>(\log T)^{B}}} P_{\Lambda}(T)^{3} \ll \frac{T^{6}}{(\log T)^{B-1}},
$$

where the sum is over primitive T-bounded lattices $\Lambda \subset \mathbb{Z}^{3}$.

Proof. We will use summation by parts as well as Theorem 2 to bound the sum. In order to do so we will denote

$$
m_{T}(r)=\mid\left\{\Lambda \subset \mathbb{Z}^{3}, \text { 2-dim. lattice }: \operatorname{det}(\Lambda)=r,\left|\lambda_{2}\right| /\left|\lambda_{1}\right|>(\log (T))^{B}\right\} \mid \text {. }
$$


so that $\sum_{r \leq t} m_{T}(r)=N\left((\log T)^{B}, t\right)$.

Using the trivial inequality $P_{\Lambda}(T) \leq N_{\Lambda}(T) \ll \frac{T^{2}}{\operatorname{det}(\Lambda)}$, as in the proof of lemma 4, we have:

$$
\begin{aligned}
& \sum_{\substack{\operatorname{det}(\Lambda) \leq \frac{T^{2}}{(\log T)^{A}} \\
\frac{\left|\lambda_{2}\right|}{\left|\lambda_{1}\right|}>(\log T)^{B}}}\left(P_{\Lambda}(T)\right)^{3} \ll T^{6} \cdot \sum_{\substack{\operatorname{det}(\Lambda) \leq \frac{T^{2}}{(\log T)^{A}} \\
\frac{\left|\lambda_{2}\right|}{\left|\lambda_{1}\right|}>(\log T)^{B}}} \frac{1}{(\operatorname{det}(\Lambda))^{3}} \\
= & T^{6} \cdot \sum_{r=1}^{\left\lfloor\frac{T^{2}}{(\log T)^{A}}\right\rfloor} \frac{m_{T}(r)}{r^{3}} \ll T^{6} \cdot \sum_{r=2}^{\left\lfloor\frac{T^{2}}{(\log T)^{A}}\right\rfloor} \frac{m_{T}(r)}{r^{3}} \\
\ll & T^{6} \cdot\left[\left.\frac{N\left((\log T)^{B}, t\right)}{t^{3}}\right|_{2} ^{\frac{T^{2}}{(\log T)^{A}}}+\int_{2}^{\frac{T^{2}}{(\log T)^{A}}} \frac{N\left((\log T)^{B}, t\right)}{t^{4}} d t\right]
\end{aligned}
$$

By Theorem 2, this is $\ll T^{6} /(\log T)^{B-1}$ as required.

As in the case of lemma 4, we proved here also:

$$
\sum_{\substack{\operatorname{det}(\Lambda)<\frac{T^{2}}{(\log T)^{A}} \\ \frac{\left|\lambda_{2}\right|}{\left|\lambda_{1}\right|}>(\log T)^{B}}} c(T, \Lambda) \ll \frac{T^{6}}{(\log T)^{B-1}},
$$

Lemma 6. Let $\Lambda \subset \mathbb{Z}^{3}$ be a 2-dimensional lattice, with a reduced basis $\left\{\lambda_{1}, \lambda_{2}\right\}$, such that $\operatorname{det}(\Lambda) \leq \frac{T^{2}}{(\log T)^{A}}$ and $\frac{\left|\lambda_{2}\right|}{\left|\lambda_{1}\right|} \leq(\log T)^{B}$. Then

$$
\frac{T \log T}{\left|\lambda_{1}\right|} \ll \frac{T^{2}}{\operatorname{det}(\Lambda)} \cdot \frac{1}{(\log T)^{\frac{A-B}{2}-1}}
$$

Proof.

$$
\left|\lambda_{2}\right|=\sqrt{\frac{\left|\lambda_{2}\right|}{\left|\lambda_{1}\right|} \cdot\left(\left|\lambda_{1}\right|\left|\lambda_{2}\right|\right)} \ll \sqrt{(\log T)^{B} \cdot \operatorname{det}(\Lambda)} \ll \frac{T}{(\log T)^{\frac{A-B}{2}}} .
$$

Therefore,

$$
\begin{aligned}
\frac{T \log T}{\left|\lambda_{1}\right|} & =\frac{T \log T}{\left|\lambda_{1}\right|\left|\lambda_{2}\right|} \cdot\left|\lambda_{2}\right| \ll \frac{T \log T}{\operatorname{det}(\Lambda)} \cdot \frac{T}{(\log T)^{\frac{A-B}{2}}} \\
& =\frac{T^{2}}{\operatorname{det}(\Lambda)} \cdot \frac{1}{(\log T)^{\frac{A-B}{2}-1}},
\end{aligned}
$$

which concludes the proof of the lemma. 
We will always want to choose the constants $\mathrm{A}$ and $\mathrm{B}$, for which $\frac{A-B}{2}-$ $1>0$, since in this case the error term of certain counting function will be asymptotically less than the corresponding main term, as we will notice in the following corollary, which follows immediately from the previous lemma. In fact, we would like to choose constants, that will satisfy

$$
\frac{A-B}{2}-1 \geq 1, A>0, B>1
$$

for example, $\mathrm{A}=6, \mathrm{~B}=2$, so this error term will not affect the general error term.

Using the proof of lemma 8 below with case (ii) of lemma 2 and substituting the result of lemma [6] we obtain:

Corollary 1. Let $\lambda \in \mathbb{Z}^{3}$ with $|\lambda| \leq \frac{T^{2}}{(\log T)^{A}}$, such that $\frac{\left|\lambda_{2}\right|}{\left|\lambda_{1}\right|} \leq(\log T)^{B}$, for constants $A, B$, which satisfy (18). Then, under the notations of (12),

$$
\epsilon(T, \lambda) \ll c(T, \lambda) \cdot \frac{1}{(\log T)^{\frac{A-B}{2}-1}}=o(c(T, \lambda)) .
$$

We are now ready to finish the proof of case (ii) of theorem 11.

Recall (11) and choose the constants $A, B$, which satisfy (18). We will ignore the difference between $\ll$ and $\leq$, which is not significant for bounding the error terms as well as computation of the main term, as we will see a bit later. Thus:

$$
\begin{aligned}
& \sum_{|\lambda| \leq T^{2}}^{\prime}\left|P A_{\lambda}(T)\right|=\sum_{|\lambda| \leq T^{2}}^{\prime}\left(P_{\lambda^{\perp}}(T)\right)^{3} \\
& =\sum_{|\lambda| \leq \frac{T^{2}}{(\log T)^{A}}}^{\prime}\left(P_{\lambda^{\perp}}(T)\right)^{3}+\sum_{\frac{T^{2}}{(\log T)^{A}}<|\lambda| \leq T^{2}}^{\prime}\left(P_{\lambda^{\perp}}(T)\right)^{3} \\
& =\sum_{\substack{|\lambda| \leq \frac{T^{2}}{(\log T)^{A}} \\
\frac{\left|\lambda_{2}\right|}{\left|\lambda_{1}\right|} \leq(\log T)^{B}}}^{\prime}\left(P_{\lambda^{\perp}}(T)\right)^{3}+\sum_{\substack{|\lambda| \leq \frac{T^{2}}{(\log T)^{A}} \\
\frac{\left|\lambda_{2}\right|}{\left|\lambda_{1}\right|}>(\log T)^{B}}}^{\prime}\left(P_{\lambda^{\perp}}(T)\right)^{3}+O\left(T^{6} \log (\log T)\right) \\
& =\sum_{|\lambda| \leq \frac{T^{2}}{(\log T)^{A}}}^{\prime}\left(P_{\lambda^{\perp}}(T)\right)^{3}+O\left(T^{6} \log (\log T)\right) . \\
& \frac{\left|\lambda_{2}\right|}{\left|\lambda_{1}\right|} \leq(\log T)^{B}
\end{aligned}
$$

We used here lemmas 4 and 5 (recall that $B>1$ because of (18) $)$. 
Substituting the result of corollary 1 into the last sum we get:

$$
\begin{aligned}
& \sum_{\substack{|\lambda| \leq \frac{T^{2}}{\left|\lambda_{2}\right| \leq(\log T)^{A}} \\
\left|\lambda_{1}\right| \leq(\log T)^{B}}}^{\prime}\left(P_{\lambda^{\perp}}(T)\right)^{3}=\sum_{\substack{|\lambda| \leq \frac{T^{2}}{(\log T)^{A}} \\
\frac{\left|\lambda_{2}\right|}{\left|\lambda_{1}\right|} \leq(\log T)^{B}}}^{\prime}(c(T, \lambda))\left(1+O\left(\frac{1}{(\log T)^{\frac{A-B}{2}-1}}\right)\right) \\
& =\left(1+O\left(\frac{1}{(\log T)^{\frac{A-B}{2}-1}}\right)\right) \cdot \sum_{|\lambda| \leq \frac{T^{2}}{(\log T)^{A}}}^{\prime}(c(T, \lambda)) \\
& \frac{\left|\lambda_{2}\right|}{\left|\lambda_{1}\right|} \leq(\log T)^{B} \\
& =\left(1+O\left(\frac{1}{(\log T)^{\frac{A-B}{2}-1}}\right)\right) \cdot \sum_{|\lambda| \leq \frac{T^{2}}{(\log T)^{A}}}^{\prime}(c(T, \lambda))+O\left(T^{6}\right) \\
& =\left(1+O\left(\frac{1}{(\log T)^{\frac{A-B}{2}-1}}\right)\right) \cdot \sum_{|\lambda| \leq T^{2}}^{\prime}(c(T, \lambda))+O\left(T^{6} \log (\log T)\right) .
\end{aligned}
$$

We used here corollary 11 as well as (15) and (16). It should be noted, that usage of lemmas 4 and 5 as "black boxes" is not enough in this case, since each summand of the current series is not necessarily less or equal to the corresponding one in these lemmas (because of the error term). Substituting (12) we obtain (writing « rather than $\leq$ in the domain of summation for consistency with (11)).

$$
P N_{3}(T) \sim \sum_{|\lambda| \ll T^{2}}^{\prime}(c(T, \lambda))=\frac{T^{6}}{\zeta(2)^{3}} \cdot \sum_{|\lambda| \ll T^{2}}{ }^{\prime} \frac{v_{2}^{3}}{|\lambda|^{3}}
$$

It remains to compute the inner sum. The same computation holds for $n \geq 4$ and will be used in section 5, so we will state it for general $n$.

\section{Lemma 7.}

$$
\sum_{|\lambda| \leq M} \frac{v_{n-1}^{n}}{|\lambda|^{n}}=\frac{2 \cdot u_{n}}{\zeta(n)} \log (M)+O(1)
$$

Proof of lemma 7 . Using Moebius inversion on the set of multiples of each primitive vector separately, we obtain:

$$
\sum_{|\lambda| \leq M} \frac{v_{n-1}^{n}}{|\lambda|^{n}}=\sum_{k=1}^{M} \mu(k) k^{-n} \sum_{1 \leq|u| \leq\lfloor M / k\rfloor} \frac{v_{n-1}^{n}}{|u|^{n}}
$$

Thus, just as in [K], we get:

$$
\sum_{|\lambda| \leq M} \frac{v_{n-1}^{n}}{|\lambda|^{n}}=\sum_{k=1}^{M} \mu(k) k^{-n} v_{n-1}^{n} \int_{1 \leq|x| \leq M / k} \frac{d x}{|x|^{n}}+O(1) .
$$


The reason that the last equality holds is that

$$
\begin{aligned}
\left|\sum_{1 \leq|u| \leq M / k} \frac{v_{n-1}^{n}}{|u|^{n}}-\int_{1 \leq|x| \leq M / k} \frac{d x}{|x|^{n}}\right| & \ll \int_{1 \leq|x| \leq M / k} \frac{d x}{|x|^{n+1}} \\
& \leq \int_{|x| \geq 1} \frac{d x}{|x|^{n+1}}<\infty
\end{aligned}
$$

and the fact that the series $\sum_{k=1}^{\infty} \mu(k) k^{-n}$ converges absolutely. Computing the last integral in (22) in polar coordinates we get:

$$
\begin{aligned}
\sum_{|\lambda| \leq M} \frac{v_{n-1}^{n}}{|\lambda|^{n}} & \sim \sum_{k=1}^{M} \mu(k) k^{-n} \cdot 2 u_{n} \cdot(\log (M)-\log k) \\
& =\frac{2 \cdot u_{n}}{\zeta(n)} \log (M)+O(1)
\end{aligned}
$$

with $u_{n}=\frac{v_{n-1}^{n}}{2} \int_{S^{n-1}} d x$, where the last integral is computed in the usual spherical [polar] coordinates. Thus, using the formula for $v_{n}$ as well as the fact that $v_{n}=\frac{1}{n} \int_{S^{n-1}} d x$, yields (2).

Thus,

$$
\sum_{|\lambda| \leq M} \frac{v_{n-1}^{n}}{|\lambda|^{n}} \sim \frac{2 \cdot u_{n}}{\zeta(n)} \log M,
$$

where the error term is $\mathrm{O}(1)$, which yields (20) and completes the proof of lemma 7

Substituting the result of the last lemma in (19) with $M=c_{n} \cdot T^{2}$, where $c_{n}$ is the constant implied by the "«"-notation in (11), will yields case (ii)] of theorem 11. The error term $O(1)$ does not bother us, since after multiplying it by const $\cdot T^{n^{2}-n}$, while substituting it in (11), we will get an error term of $O\left(T^{n^{2}-n}\right)$, and adding it to other error terms will not increase an estimate for the general error term of the asymptotics. As mentioned before, the constant $c_{n}$ does not affect the computation, since we substitute it in a logarithm in any case.

\section{The case $n \geq 4$.}

We will need the following lemma: 
Lemma 8. Let $\lambda \in \mathbb{Z}^{n}$ be a primitive vector, such that its orthogonal dual, $\lambda^{\perp}$ is T-bounded. Let $\left\{\lambda_{1}, \lambda_{2}, \ldots, \lambda_{n-1}\right\}$ be a reduced basis of $\lambda^{\perp}$. Then for $n \geq 4$ we have

$$
\begin{aligned}
\left|P A_{\lambda}(T)\right| & =\frac{v_{n-1}^{n}}{\zeta^{n}(n-1)|\lambda|^{n}} T^{n^{2}-n} \\
& +O\left(\frac{T^{n^{2}-n-1}}{\left|\lambda_{1}\right|^{n} \cdot\left|\lambda_{2}\right|^{n} \cdot \ldots \cdot\left|\lambda_{n-2}\right|^{n} \cdot\left|\lambda_{n-1}\right|^{n-1}}\right) .
\end{aligned}
$$

Proof. Due to (9), we have $\left|P A_{\lambda}(T)\right|=\left(P_{\lambda^{\perp}}(T)\right)^{n}$, and substituting the case (i) of lemma 2 in the last equality, as well as the fact that $\operatorname{det}\left(\lambda^{\perp}\right)=|\lambda|$, because of (7), we get: $\left|P A_{\lambda}(T)\right|=\left(\frac{v_{n-1}}{\zeta(n-1)|\lambda|} T^{n-1}+O\left(\frac{T^{n-2}}{\left|\lambda_{1}\right| \cdot\left|\lambda_{2}\right| \cdot \ldots \cdot\left|\lambda_{n-2}\right|}\right)\right)^{n}=$ $(a+b)^{n}$. We will use the binomial formula for the last expression. The first summand (that is, $a^{n}$ ) is just the main term in the result of the lemma. Now, since the lattice $\lambda^{\perp}$ is T-bounded, $a$ is asymptotically greater than $b$ (that is $a \gg b$ ), and thus the only asymptotically significant summand in the binomial is the second one (that is $n \cdot a^{n-1} b$ ). The coefficient $n$ is constant, and thus, by (66), this is the error term we just stated in the lemma.

Notations: In this section we will use the notations in (13) as well.

Substituting the result of Lemma 8 into (11), we obtain:

$$
\begin{aligned}
P N_{n}(T) & =\frac{1}{2} \cdot \sum_{|\lambda| \ll T^{n-1}}^{\prime}\left(\frac{v_{n-1}^{n}}{\zeta^{n}(n-1)|\lambda|^{n}} T^{n^{2}-n}+\epsilon(T, \lambda)\right)+O\left(T^{n^{2}-n}\right) \\
& =\frac{T^{n^{2}-n}}{2 \cdot \zeta^{n}(n-1)} \cdot \sum_{|\lambda| \ll T^{n-1}} \frac{v_{n-1}^{n}}{|\lambda|^{n}}+\epsilon_{2}^{\prime}(T)+O\left(T^{n^{2}-n}\right),
\end{aligned}
$$

with

$$
\epsilon_{2}^{\prime}(T) \ll \sum_{|\lambda| \leq T^{n-1}}^{\prime}|\epsilon(T, \lambda)| .
$$

We will prove in the end of the section that $\epsilon_{2}^{\prime}(T) \ll T^{n^{2}-n}$.

Using (20) with $M=c_{n} \cdot T^{n-1}$, (where $c_{n}$ is the constant implied by the "«"-notation in (11)) in (24) will imply

$$
P N_{n}(T)=\frac{(n-1) u_{n}}{\zeta(n) \zeta(n-1)^{n}} T^{n^{2}-n} \log T+O\left(T^{n^{2}-n}\right)
$$

which ends the proof of theorem 1 [i)] 
Just as in case $n=3$, the error term $O(1)$ does not bother us. Thus, $P N_{n}(T)=\frac{(n-1) u_{n}}{\zeta(n) \zeta(n-1)^{n}} T^{n^{2}-n} \log T+\epsilon^{\prime}(T)$, where $\epsilon^{\prime}(T)$ is the error term of this asymptotics. Accumulating all the error terms we confronted with and assuming $\epsilon_{2}^{\prime}(T) \ll T^{n^{2}-n}$ (which we will prove immediately), will imply $\left|\epsilon^{\prime}(T)\right| \ll T^{n^{2}-n}$.

Error term: The only error term which is not less or equal to the corresponding error term in $[\mathrm{K}]$ is $\epsilon_{2}^{\prime}$. However in this case, we can immediately bound it given the results of the work that was already done by Y. Katznelson.

Under the notations (13), for $n \geq 4$ we have, due to (25) and lemma 8 .

$$
\epsilon_{2}^{\prime}(T) \ll \sum_{|\lambda| \leq T^{n-1}}^{\prime}|\epsilon(T, \lambda)| \ll \sum_{|\lambda| \leq T^{n-1}}^{\prime} \frac{T^{n^{2}-n-1}}{\left|\lambda_{1}\right|^{n} \cdot\left|\lambda_{2}\right|^{n} \cdot \ldots \cdot\left|\lambda_{n-2}\right|^{n} \cdot\left|\lambda_{n-1}\right|^{n-1}} .
$$

From the definition of $\sum^{\prime}$ and $\sum^{\prime \prime}$, it is obvious that $\sum^{\prime \prime} \leq \sum^{\prime}$ as long as only nonnegative numbers are involved. The inequality

$$
\sum_{|\lambda| \leq T^{n-1}} " \frac{T^{n^{2}-n-1}}{\left|\lambda_{1}\right|^{n} \cdot\left|\lambda_{2}\right|^{n} \cdot \ldots \cdot\left|\lambda_{n-2}\right|^{n} \cdot\left|\lambda_{n-1}\right|^{n-1}} \ll T^{n^{2}-n}
$$

was showed in [K] (pages 130-133) in the course of proving that $\epsilon_{2}(T) \ll$ $T^{n^{2}-n}$, where $\epsilon_{2}(T)$ is the corresponding error term in the case of $N_{n}(T)$.

\section{References}

[FMT] Jens Franke, Yuri I. Manin and Yuri Tschinkel, Rational points of bounded height on Fano varieties. Invent. Math. 95 (1989), no. 2, 421435.

[K] Yonathan R. Katznelson. Singular Matrices and a Uniform Bound for Congruence Groups of $S L_{n}(\mathbb{Z})$. Duke Mathematical Journal, 1993, Vol. 69, No. 1, pages 121-136

[SCH] Wolfgang M. Schmidt. Asymptotic Formulae for Point Lattices of Bounded Determinant and Subspaces of Bounded Height. Duke Mathematical Journal, 1968, No. 35, pages 327-339

[SCD] Wolfgang M. Schmidt. The Distribution of Sublattices of $\mathbb{Z}^{m}$. Monatshefte fur Mathematik, 1998, Vol. 125, pages 37-81

[SGL] Carl Ludwig Siegel. Lectures on the Geometry of Numbers. SpringerVerlag, Berlin, 1988 